Review Article

\title{
Validity of Accelerometers for the Evaluation of Energy Expenditure in Obese and Overweight Individuals: A Systematic Review
}

\author{
Silvia Pisanu $\left(\mathbb{D},{ }^{1}\right.$ Andrea Deledda, ${ }^{2}$ Andrea Loviselli $\mathbb{D},{ }^{2}$ Inge Huybrechts $\mathbb{D},{ }^{3}$ \\ and Fernanda Velluzzi ${ }^{2}$ \\ ${ }^{1}$ Department of Biomedical Sciences, University of Cagliari, Cagliari, Italy \\ ${ }^{2}$ Department of Medical Sciences and Public Health, University of Cagliari, Cagliari, Italy \\ ${ }^{3}$ International Agency for Research on Cancer, Nutrition and Metabolism Section, Lyon, France
}

Correspondence should be addressed to Andrea Loviselli; alovise2@gmail.com

Received 21 October 2019; Accepted 16 May 2020; Published 4 August 2020

Academic Editor: Luigi Schiavo

Copyright (C) 2020 Silvia Pisanu et al. This is an open access article distributed under the Creative Commons Attribution License, which permits unrestricted use, distribution, and reproduction in any medium, provided the original work is properly cited.

Objective. Even though the validity of accelerometers for the measurement of energy expenditure (EE) has been demonstrated for normal-weight individuals, the applicability of this instrument in obese individuals remains controversial. This review aims to summarize the level of agreement between accelerometers and the gold standards (indirect calorimetry and doubly labelled water) for the measurement of energy expenditure (EE) in obese or overweight individuals. Methods. The literature search was limited to comparison studies assessing agreement in EE determination between accelerometers and indirect calorimetry (IC) or doubly labelled water (DLW). We searched in PubMed and in Scopus until March 1, 2019. The analysis was restricted to obese or overweight adult individuals. The following descriptive information was extracted for each study: sample size, characteristics of participants (sex, age, BMI, fat mass percentage, any pathological conditions, modality of recruitment in the study, and exclusion criteria), accelerometer description (model, type and body position), and type of gold standard and validity protocol (duration, conditions, and requirements during and before the experiment). Three review authors independently screened the obtained results, and the quality of the selected articles was assessed by the QUADAS-2 tool. Results. We obtained seventeen eligible articles, thirteen of which showed concerns for the applicability section, due to the patient selection. Regarding the accelerometers, nine devices were validated in the included studies with the BodyMedia SenseWear ${ }^{\circledR}$ (SWA) being the most frequently validated. Although correlations between accelerometers and the gold standard were high in some studies, agreement between the two methods was low, as shown by the Bland-Altman plots. Conclusions. Most accelerometer estimations of EE were inaccurate for obese/overweight subjects, and authors advise to improve the accuracy of algorithms for SWA software, or the predicted equations for estimating EE from other accelerometers.

\section{Background}

The assessment of energy expenditure (EE) is essential both in healthy individuals, such as sporty people, and in clinical studies, for the establishment of the amount of physical activity associated with energy balance, fitness, and health benefits [1]. Furthermore, the knowledge of EE plays a central role in the evaluation and management of all conditions that require weight loss or weight maintenance, like obesity [2], and in all the clinical conditions in which physical activity might have a therapeutic value, like hypertension, stroke, coronary heart disease, type I and type II diabetes, metabolic syndrome, and cancer. Given that the fat loss induced by a certain physical exercise has a great individual variability [3], a correct measurement of EE supports a personalized management of weight loss. The physical fitness assessment of overweight and obese patients is fundamental, considering the important benefits of physical exercise as therapy in this population, not only to counteract the cardiovascular risk but also for maintaining 
the muscle tone, increasing the metabolic rate, and decreasing the risk of common chronic diseases [4].

The traditional gold standard method, the direct calorimetry, has been largely replaced by indirect calorimetry, due to its practicality and cost-effective use. The indirect calorimetry (IC) and the doubly labelled water (DLW) method are the most commonly used gold standards for determining EE $[5,6]$. The DLW technique is suitable for use in free-living contexts and provides an accurate measure of total EE (TEE). However, the cost and the requirement of isotope ratio mass spectrometry for analysis prohibit DLW from being used in large population studies. Furthermore, this technique provides an accurate measure of TEE, but no information on physical activity (PA) patterns in terms of frequency, duration, intensity, and energy expenditure is available [6].

IC assesses the amount of heat generated by the oxidation of food substrates, which are converted into $\mathrm{CO}_{2}$, $\mathrm{H}_{2} \mathrm{O}$, and heat. Specifically, EE is calculated by measuring the amount of oxygen used, and carbon dioxide released by the body [5, 7]. The IC allows for real-time measurement of PA, adding the dimensions of duration and intensity. However, the limited access to the equipment and the technical knowledge required for supervision limit the use of portable IC for true field settings or for studies on large populations [8].

The accelerometer represents a valid, noninvasive method for measuring PA under free-living conditions. Accelerometers are designed as small, lightweight, unobtrusive portable devices, with very low operating costs, able to assess PA [9]. Commercial accelerometers usually convert the magnitude of accelerations to provide "activity counts" per defined period of time (epoch). The activity counts represent the estimated intensity of measured activities during each time period. Several regression equations can be derived or validated for different accelerometers to better match the exact EE of physical activities among individuals [10]. Uniaxial accelerometers measure accelerations in one direction, usually in the vertical plane, whereas triaxial accelerometers measure accelerations in the anteroposterior, mediolateral, and vertical direction [11].

The BodyMedia SenseWear ${ }^{\circledR}$ Armband (SWA) is a sleek, wireless, and wearable body monitor that enables continuous physiological monitoring outside the laboratory [12].

The SWA is worn on the posterior side of the master arm and uses a unique combination of sensors. A sensor that detects the heat flow measures the amount of heat dissipated by the body. The skin temperature and the temperature near the instrument are measured by sensitive thermistors. The device also measures the galvanic skin response, which varies with physical and emotional stimuli. An accelerometer follows the movements of the arm and provides information on body position. Individual baseline data, i.e., age, sex, weight, and height, have to be inserted for allowing the activation of the device [12]. Having more sensors is very important for the ability of SWA to accurately monitor physiological conditions. In fact, the presence of multiple sensors allows for the disambiguation of the contexts that could confuse a single sensor. For example, if the movement of a person is high, it may be caused by an exercise or be due to being in a vehicle in movement. However, the variations in temperature, sweat, and heat flow are generally very different for these two situations [9]. The software algorithms use the physiological signals of all sensors to detect the context and then apply the correct formula for the estimation of energy consumption based on sensor values. SWA is able to recognize many basic activities, such as lifting weights, walking, running, cycling, resting, and going by car, bus, or train. Other activities are classified as combinations of these basic activities. The sequential release of software (i.e., version 5.0, 6.1, 7.0, and 8.0) included refined algorithms (i.e., v 2.0, 2.2, and 5.0) designed to improve accuracy and utility [13].

Although the reliability of SWA for the measurement of both REE and EE during physical exercises or in free-living conditions has been demonstrated in different studies considering normal-weight healthy individuals, its applicability remains controversial [14-20]. Under free-living conditions, SWA (software versions 6.1 and 7.0) demonstrated a good agreement with DLW in the Bland-Altman plot and high values of intraclass correlation coefficient (ICC) (>0.80) in healthy subjects [16]. Similarly, laboratory experiments demonstrated reliable estimates of EE (no significant differences versus IC in mean $\pm S D$, or correlations with IC estimates between 0.47 and 0.69) [19, 20]. However, Zorrilla-Revilla et al. found an important overestimation (41.31\%) of EE when walking carrying load, while Tucker et al. obtained a significant underestimation (18\%) of EE in multiple trials (SWA version 7.0 in both the studies). Regarding its accuracy for the measurement of REE, in the study of Malavolti et al. on healthy subjects, Bland-Altman plot showed no difference in REE determination between SWA (version 4.0) and IC [17], and authors judged the SWA as a reliable device for measuring REE in healthy subjects. In line with these findings, in the study of Zorrilla-Revilla et al., the SWA (version 7.0) estimates of RMR in healthy adults were associated with small error scores (mean absolute percentage error $=17.31 \%$; mean difference $=11.1 \%$ ) [14].

Overweight and obesity are responsible for biomechanical modifications during walking, with loss of efficiency [21]. As a consequence of the lack of efficiency, accelerometers may overestimate EE of obese individuals due to excessive body motion (greater body movement associated with reduced mechanical efficiency) $[19,37]$. Other concerns on the applicability of accelerometers in obese individuals are the obvious differences in the placement of these devices with respect to the center of mass of the body, and the different patterns of PA in daily life. Consequently, the accelerometer output, the EE estimates that derive from it, and the accuracy of these estimates compared with criterion methods can be affected [9]. An advantage of SWA can be the potential ability to detect false motion and the detection of nonambulatory physical activity, which is provided by the combination of the accelerometer data with the other physiological sensors. However, it is possible that higher levels of body fatness may impact the accuracy of the existing algorithms [37]. 
The aim of our systematic review is to summarize the existing evidences for the level of agreement between accelerometers and gold standards (IC or DLW) for the measurement of $\mathrm{EE}$ in obese or overweight individuals.

\section{Methods}

Cohort studies, intervention studies, and validation studies were considered in our analysis. In order to be included, studies were required to report the comparison between accelerometers and gold standard results in the same individuals and in the same conditions. Our analysis was restricted to obese or overweight adult individuals (older than 18 years, BMI $\geq 25 \mathrm{~kg} / \mathrm{m}^{2}$ ). Studies on participants with illnesses or conditions that may affect EE (fever, infections, immunodeficiency syndrome, cancer, and traumatic injury) or undergoing any type of elective surgical procedures were excluded. The search was limited to publications written in English.

2.1. Search Method. The literature search was limited to method comparison studies assessing agreement in EE determination between accelerometers and IC or DLW, which we considered as gold standards [22,23]. The choice of these methods was based on their common use in clinical practice and in intervention and validity studies. We searched in PubMed and in Scopus (final search on 1 March 2019), using combinations of the following keywords: "accelerometer," "SenseWear armband," "accelerometry," "motion sensor," "activity monitor," "armband," "multi-sensor," "obese," "overweight," "obesity," "validation study," "indirect calorimetry," "double labelled water," "doubly labelled water." Our search strategy was designed to incorporate studies that included the use of both the accelerometers device and the gold standard in the same population. Three review authors independently screened the obtained results. In order to increase the sample size, each study selected for being included in the review was inserted in Google Scholar, using the function "cited by" and "correlated." The reference lists of included studies were also checked for additional relevant studies.

The eligibility of each study was initially based upon details presented in the abstract followed by reading the full text of all possible studies. Disagreements on the inclusion or exclusion of each study were resolved by consensus.

2.2. Data Extraction. Data relating to sample size, characteristics of individuals (age, sex, BMI, fat mass percentage, and any pathological conditions), exclusion criteria, accelerometers (model and location, and software or algorithm used for data analysis), gold standard method, protocol of the experiment, primary results of outcome measures, and reported statistics including statistical significance and conclusion results were extracted. Two review authors extracted the data listed above from the included studies and the third author checked the extracted data.
2.3. Study Selection Process. Our initial search yielded 343 results. After the removal of duplicates, we obtained a total of 273 articles.

The titles and/or abstracts were screened by three authors. A total of 33 publications were identified as potentially relevant according to inclusion criteria. We excluded 24 fulltext articles for the following reasons: in one study, there were no data on the accelerometer; in three studies, accelerometer and gold standard were used in different conditions; in one study, the EE from accelerometer was not calculated; in twelve studies, the accelerometer was not compared with a gold standard for the measurement of EE; in three studies, the individuals were of normal weight; in one study with a population of different BMI, this index was not considered in the results; in two studies, the mean difference between accelerometer and gold standard was not reported; and one study enrolled lactating women. Consequently, we obtained a total of 9 eligible articles. After the search performed in Google Scholar and the screening of the references of eligible studies, 8 additional articles were selected, obtaining a total of 17 included studies.

2.4. Quality Assessment. According to Whiting et al. [24], we evaluated the quality of the studies using the second version of the Quality Assessment of Diagnostic Accuracy Studies (QUADAS-2) checklist. This tool consists of four key domains: patient selection, index test, reference standard, and flow of patients through the study and timing of the index tests and reference standard ("flow and timing"). Each domain is assessed in terms of risk of bias and concerns regarding applicability. Risk of bias can be judged as "low," "high," or "unclear" based on the provided signalling questions. If all the questions for a domain are answered "yes," the risk of bias is judged as "low," while if any question is answered "no" the risk of bias is taken into consideration by the reviewers. Concerns regarding applicability are rated as "low," "high," or unclear. Unclear category is used if insufficient data were reported to permit a judgement.

\section{Results}

3.1. Characteristics of Participants in the Selected Studies. Table 1 shows the characteristics of the participants enrolled in the selected studies. The sample size ranged from 10 to 264 and the mean age ranged from 25 to 82 years ( $43 \pm 13$ years). BMI ranged from 29.2 to $43.2 \mathrm{~kg} / \mathrm{m}^{2}\left(33.4 \pm 6.7 \mathrm{~kg} / \mathrm{m}^{2}\right)$. In 8 studies, all the participants were obese or overweight $[9,25$, $30-32,37,39,40]$. In the study of Elbelt et al. [29], the population was divided into 4 groups according to BMI, the first including both normal-weight and overweight individuals. Similarly, Swartz et al. divided the sample into three groups based on the BMI category (normal weight, overweight, and obese) [37].

In the study of Correa et al., participants were recruited from two clinical trials aimed to weight loss. The first trial enrolled only participants whose BMI was $>25$ and $<40$, while the second trial enrolled participants whose BMI was $>18.5$ and $<40$ [27]. 
TABLE 1: Characteristics of participants in the included studies.

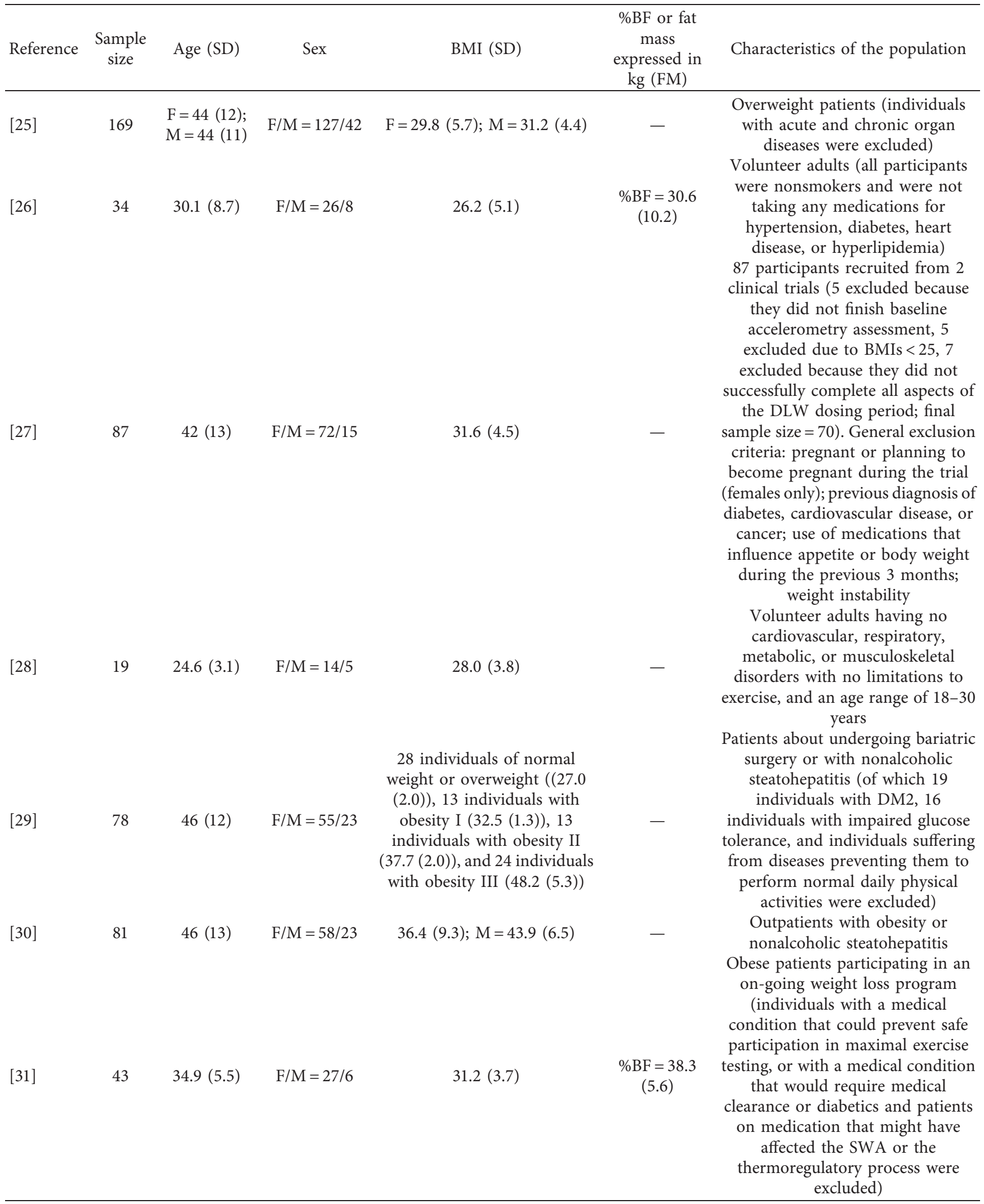


TABLE 1: Continued.

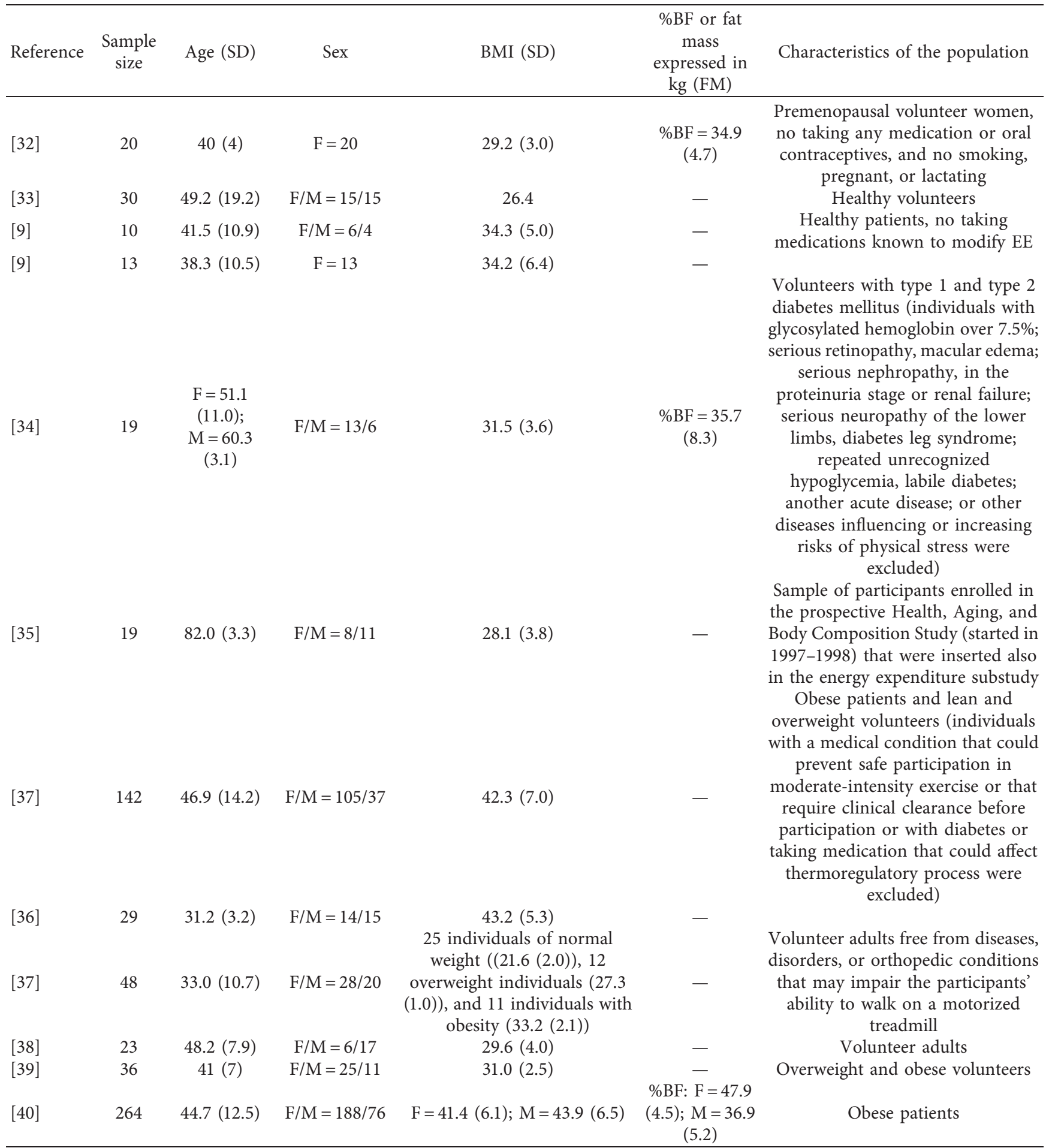

\%BF: body fat percentage; BMI: body mass index; DLW: doubly labelled water; DM2: diabetes mellitus type 2; SWA: SenseWear Armband.

Five studies recruited healthy volunteers whose mean BMI was indicative of overweight/obese status [26, 28, 33, $35,38]$.

In two studies, the population included patients with nonalcoholic steatohepatitis $[29,30]$. In addition, in the study of Machač et al., participants were volunteers with type I or type II diabetes, being obese as a group, as indicated by the mean BMI [34].

Table 2 shows the characteristics of the included studies and the validity results. 
3.2. Outcome Measures. The validity of accelerometers for the measurement of REE was evaluated in 5 studies [25, 29, $30,36,40]$. Nine studies investigated the validity of accelerometers for the measurement of PAEE, considering the execution of specific physical activities in laboratory conditions $[9,26,28,31,33,34,37,38]$, and 5 studies validated the accelerometers for the measurement of TEE or PAEE under free-living conditions [9, 27, 32, 35, 39]. All the studies used a range of statistics to assess agreement between the accelerometer and the chosen reference test. The majority of the included studies used Bland-Altman plots and Pearson correlation coefficients to show agreement and association between accelerometer and gold standard, respectively. The mean difference between accelerometer and gold standard was inserted in almost all the included studies, expressed as an absolute value or as a percentage, but the significance of the difference was not always specified. The standard deviation of the mean difference was also not always reported.

3.3. Reference Methods. For REE and PAEE, IC was used as the gold standard in all the included studies. In total, 3 and 5 different indirect calorimetry devices were employed for REE and PAEE, respectively. For the measurement of TEE or PAEE in free-living conditions, DLW was used as the gold standard in all the included studies.

3.4. Validity Protocol. Regarding accelerometers, 9 devices were validated in the included studies: SWA was the most validated, being evaluated in 11 studies using different software versions (from 4.0 to the most recent 8.1) [25-27, 29-31, 34-36, 38, 40]. The uniaxial Caltrac accelerometer was used in one study [32]; the RT3 triaxial accelerometer was used in one study, compared with the triaxial TriTracR3D [9]; one study evaluated the accuracy of the Fitbit Charge 3-axis accelerometer [28]; one study validated the Kenz Lifecorder EX accelerometer [37]; the ActiGraph GT3X + triaxial accelerometer was validated in one study [33]; one study tested the validity of the Actical omnidirectional accelerometer [27] and one study used the TracmorD triaxial accelerometer [39].

EE was assessed in laboratory during different exercise tests $[9,26,28,31,33,34,36,37]$, with IC and accelerometer at the same time. In the study of Thorp and colleagues, the accuracy of the accelerometer in estimating EE was evaluated in order to determine whether alternating bouts of sitting and standing at work influenced daily workplace EE [38]. In 4 studies, the exercise tests were performed after a fasting period of at least 2 hours [26, 31, 37, 38], and in 2 studies, abstention from physical activities for at least 3 hours before the test was required [31, 38].

3.5. Risk of Bias and Study Methodology Quality Assessment Scores. Risk of bias was low in the patient selection. However, in 8 studies [9, 25, 28, 33,34, 36, 37, 40], it was judged as high for index test and gold standard items, due to the absence of an at least 2 hours of fasting period and/or an at least 15 minutes of resting period before the test. In 5 studies, the risk of bias was judged as high for the lack of individuals included in the final analysis, due to technical problems with the accelerometers $[9,29,38]$, or because they did not finish the accelerometer assessment or the DLW dosing period [27, 35]. Referring to concerns with the applicability of the proposed test, the risk was judged as low for index test and gold standard, but the majority of studies presented an issue in the patient selection $[9,25,27-30$, 33-39] due to not having excluded smoking individuals and/ or individuals taking medication that could affect EE.

\subsection{Validity Results}

3.6.1. REE. One study obtained an underestimation of $\mathrm{REE}_{\mathrm{SWA}}$ although the statistical significance was not specified [36]. However, a significant overestimation of SWA was observed in all the other included studies [25, 30, 37, 40]. Four studies showed results of Bland-Altman plots [25, 30, $36,40]$. Pearson's correlation coefficient was reported in three studies $[29,36,40]$, in which it ranged between 0.58 (obtained in women) and 0.88 (obtained in the whole population).

In the studies of Bertoli et al. and Elbet et al., Bland-Altman plots showed a low agreement $[25,30]$. On the other hand, Papazoglou et al. reported a mean underestimation with narrower limits of agreements [36].

Results of Bland-Altman analysis revealed the tendency of the bias to increase as the REE increased across participants. Authors did not find any relationship between the bias and age, BMI, fat-free mass, total body water, and extracellular water of individuals [36], in agreement with the work of Elbelt et al., in which the bias was not significantly associated with changes in body weight [29]. In the study of Waldburger and colleagues, Bland-Altman plots indicated that SWA systematically overestimated REE in women displaying low REE values and underestimated REE in women displaying high REE values [40].

In 2012, Elbelt et al. proposed an alternative method for the evaluation of REE by SWA by measuring sleep EE (SEE) for 3 consecutive days [30]. Despite the high correlation between the two methods, the mean difference was significant, with around $6 \%$ of the included participants being outside the limits of agreements (LOA) (LOA: -715 to $-318 \mathrm{~kJ} /$ day for the early uninterrupted phase of sleep and -761 to $-377 \mathrm{~kJ} /$ day for the late uninterrupted phase of sleep) [30].

\subsection{EE during Different Physical Exercises or Sedentary} Behaviours. Five included studies presented Bland-Altman plots $[9,26,31,36,38]$, while Pearson's correlation coefficients were indicated only in three studies [31, 34, 38]. A general trend toward overestimation can be noticed (see Supplementary Material 1). However, the study protocol differs greatly among the included studies.

In the study of Papazoglou et al., the validity of SWA for the estimation of PAEE was evaluated using Inner View Research Software 4.0. for 3 different physical activities (pedalling on an ergometer, stepping, and walking on a 


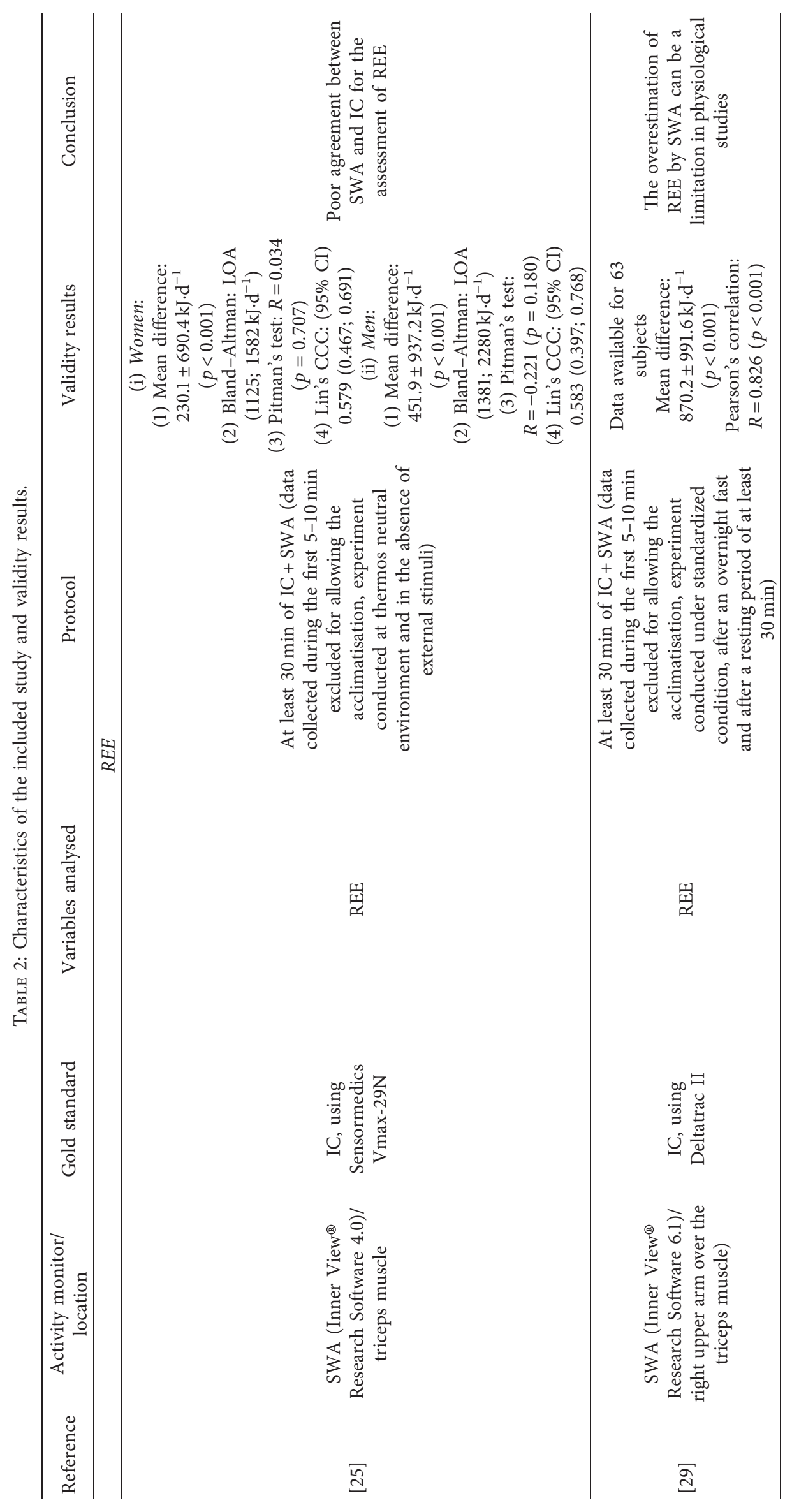




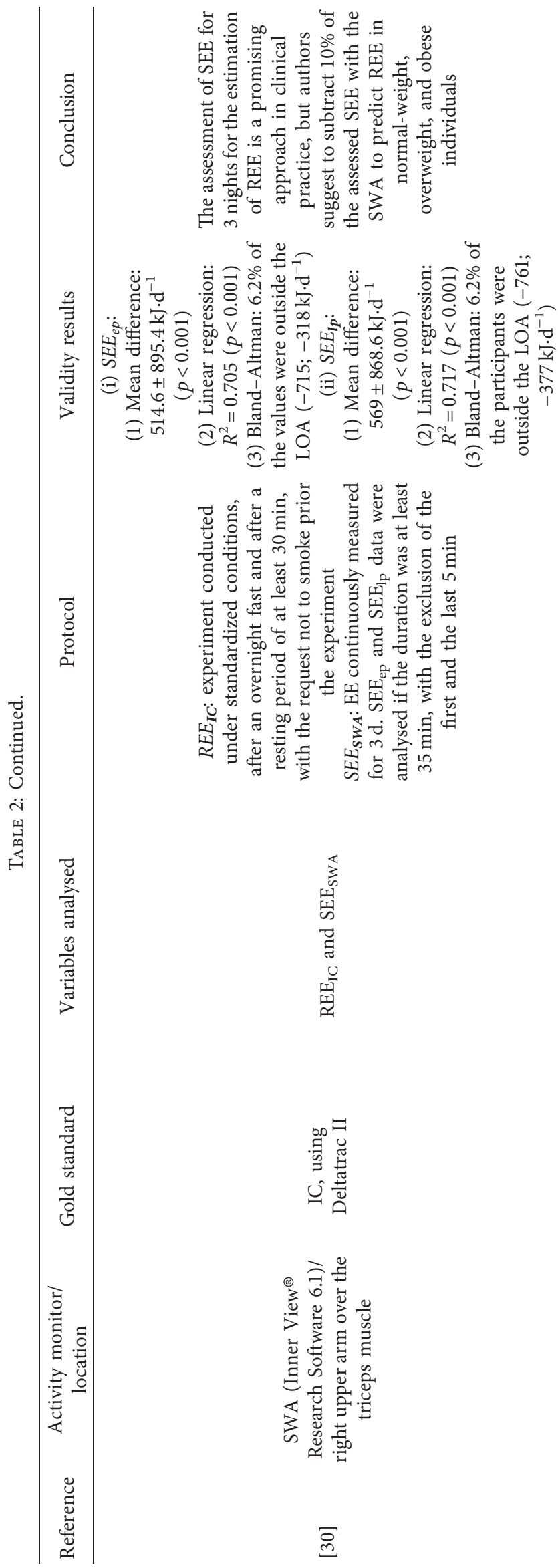

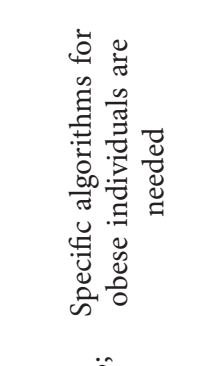

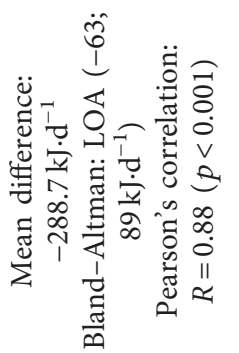

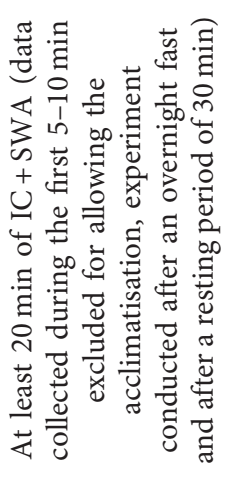

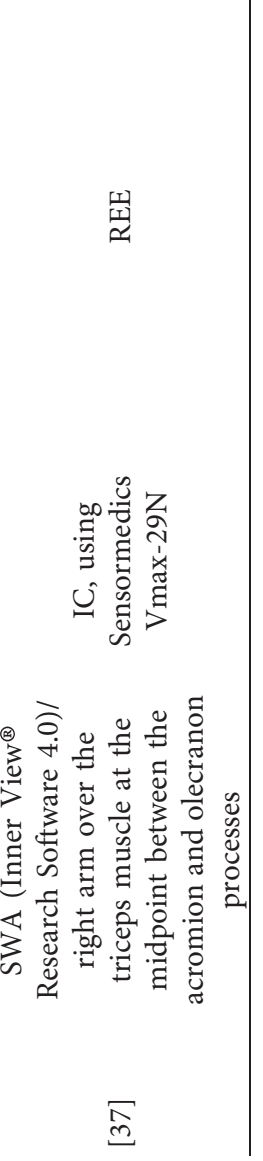

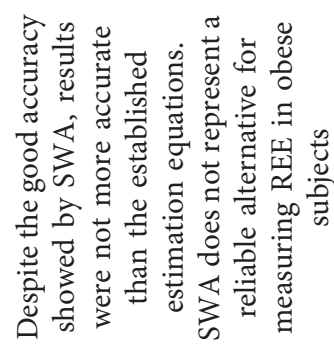

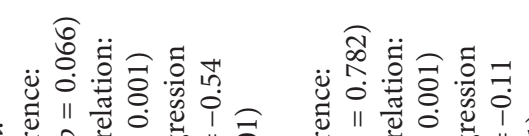

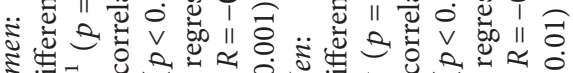

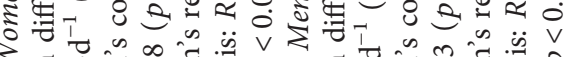

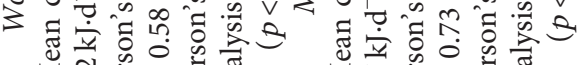

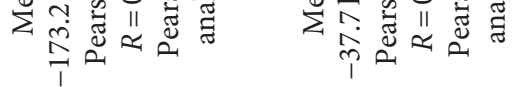

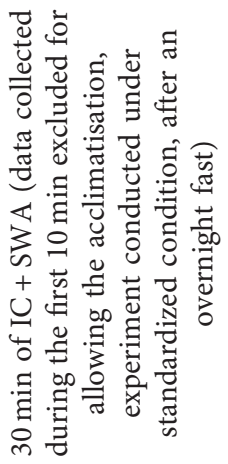

똗

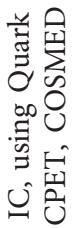

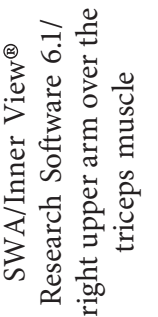

宇 


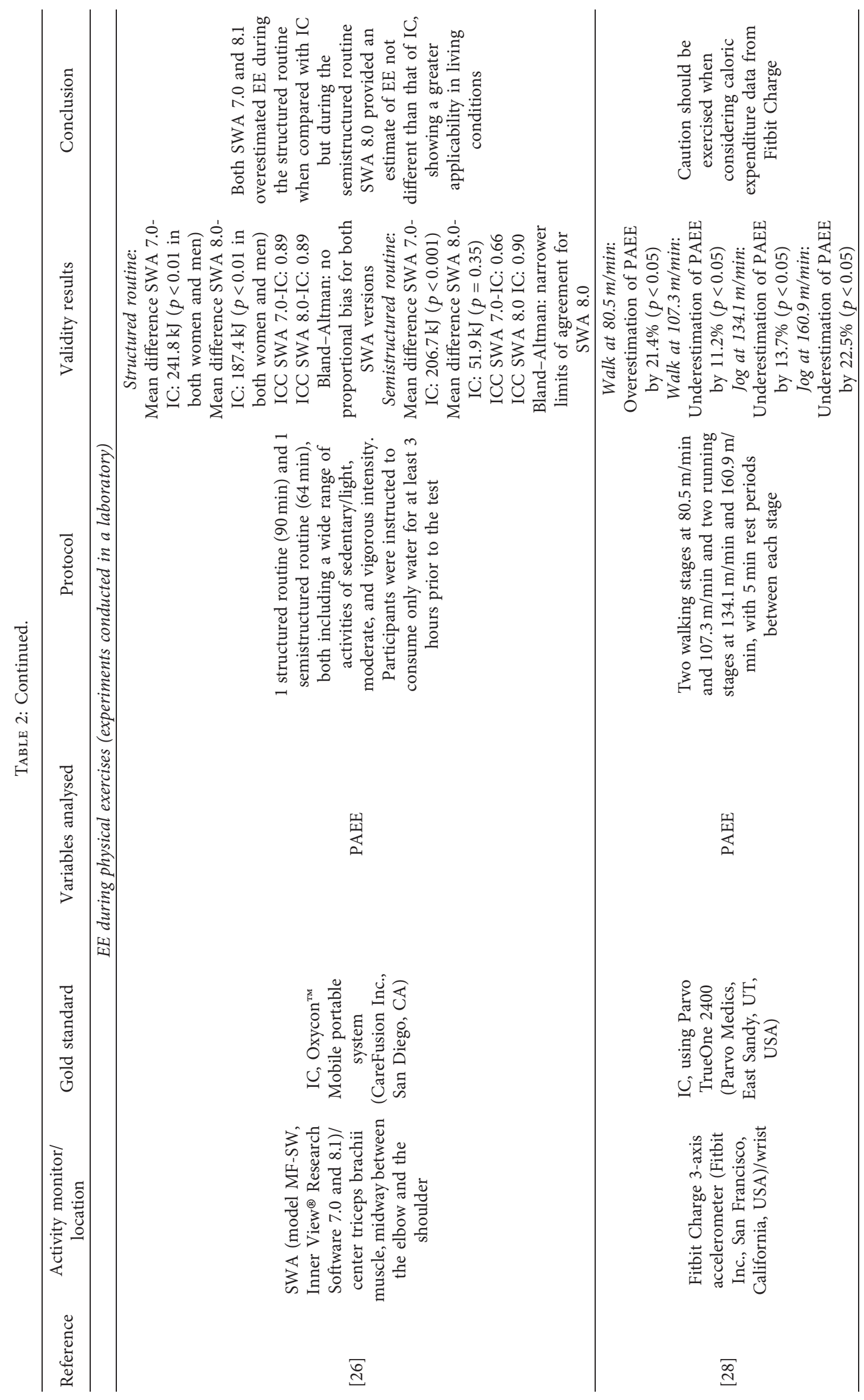




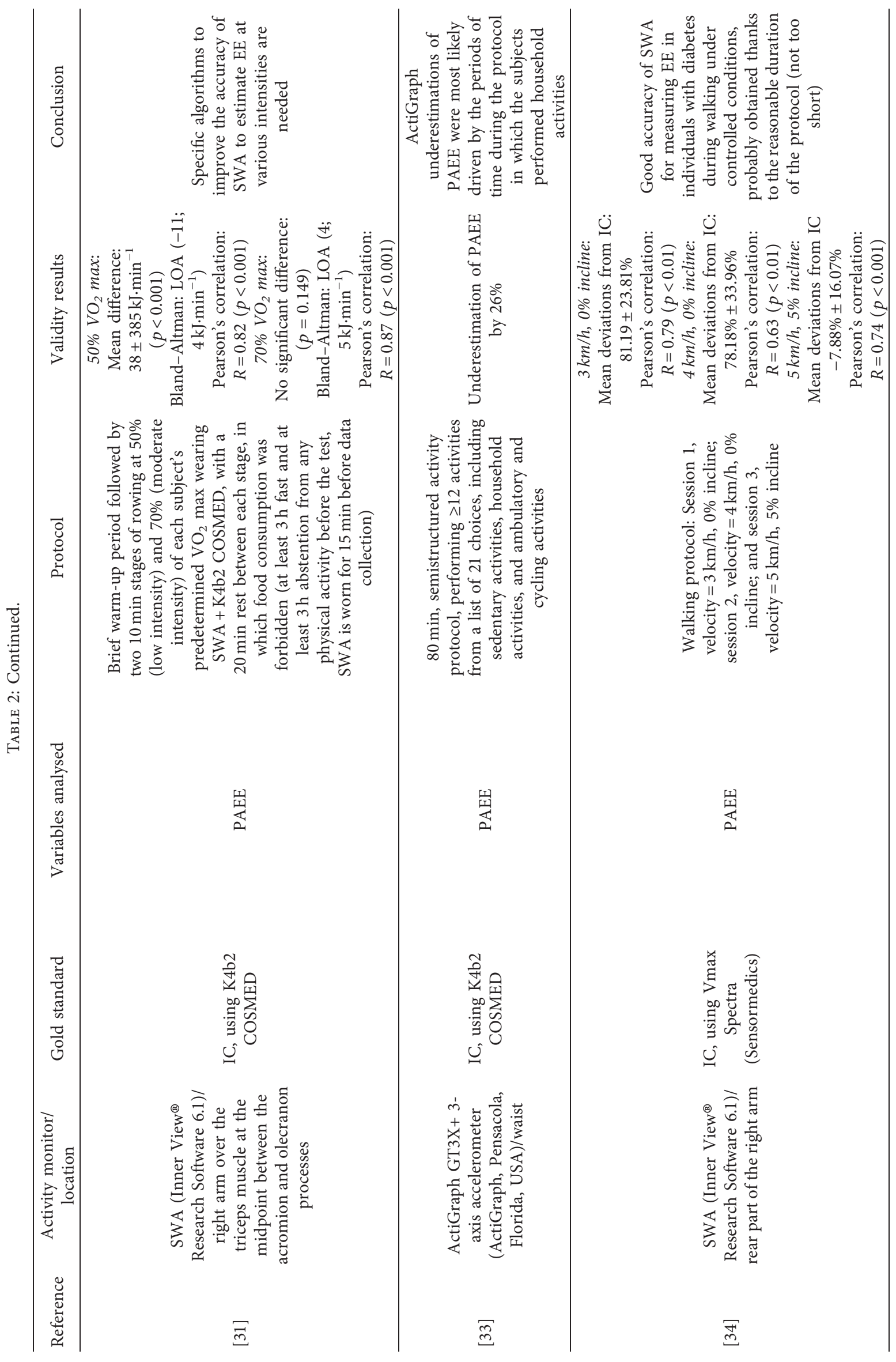




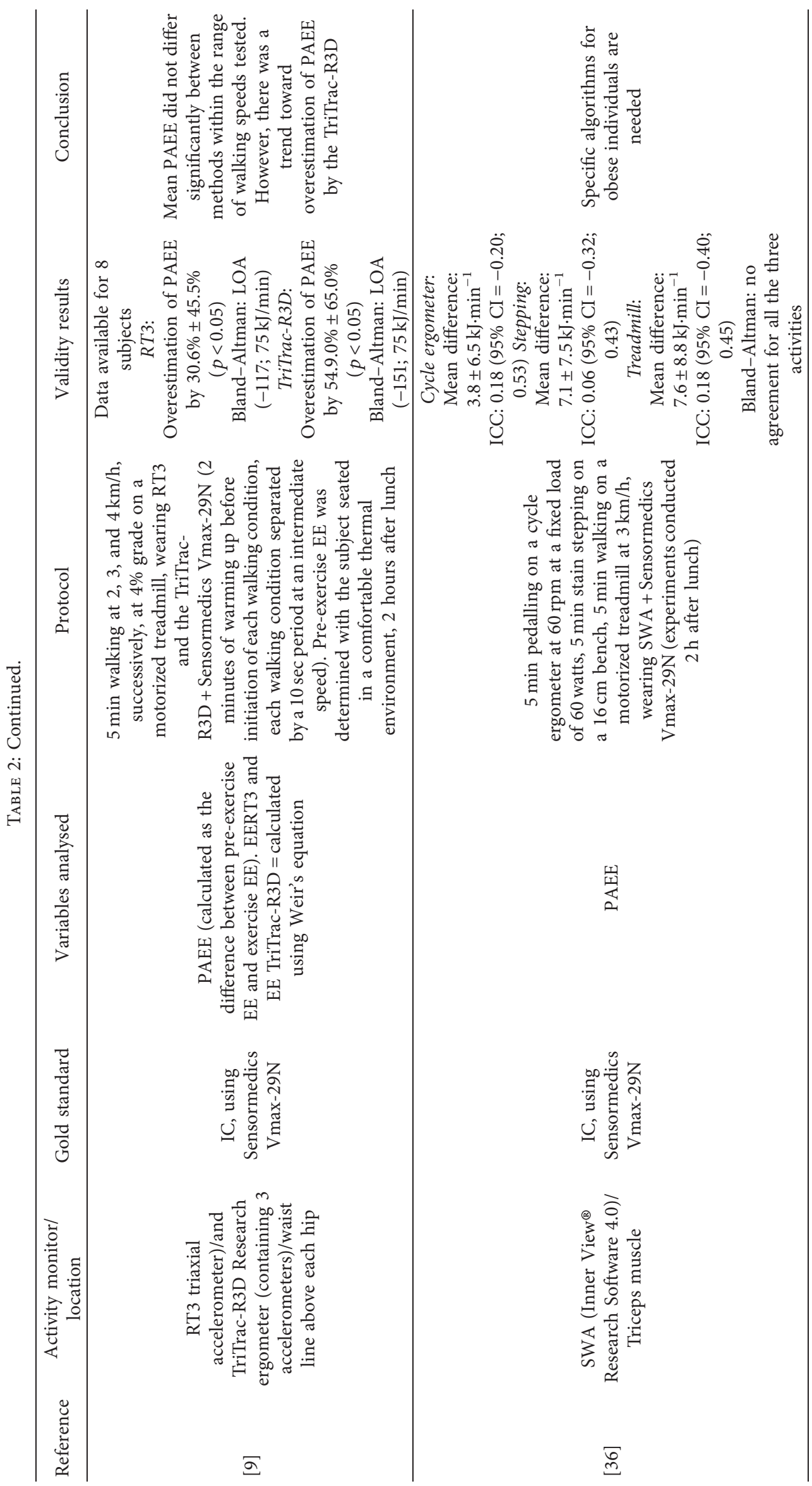




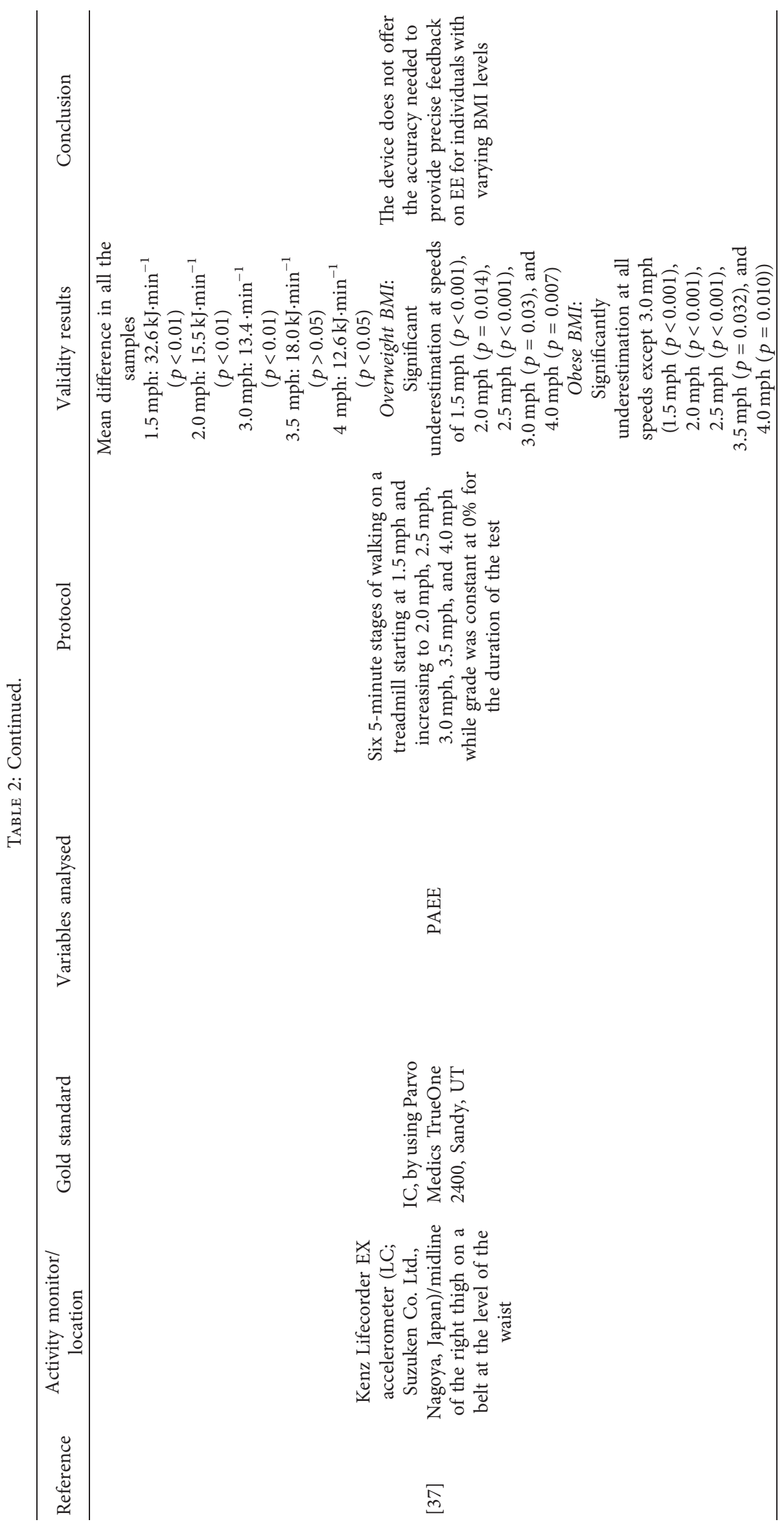




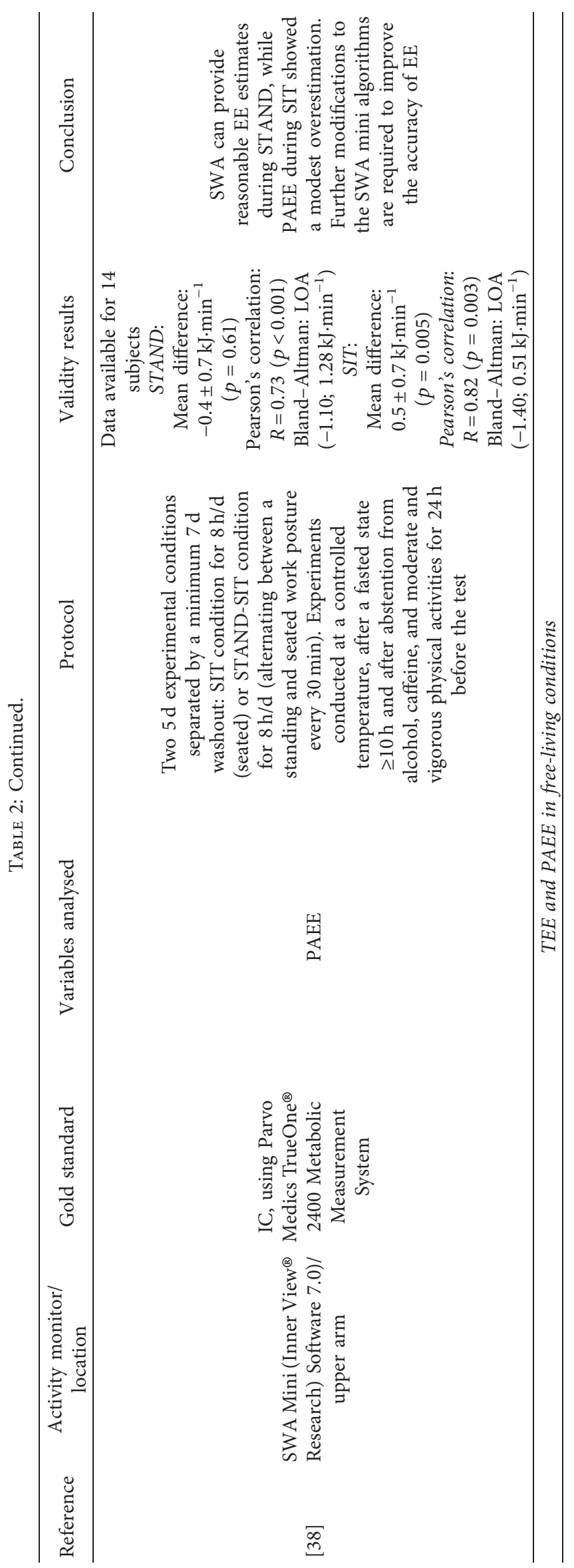




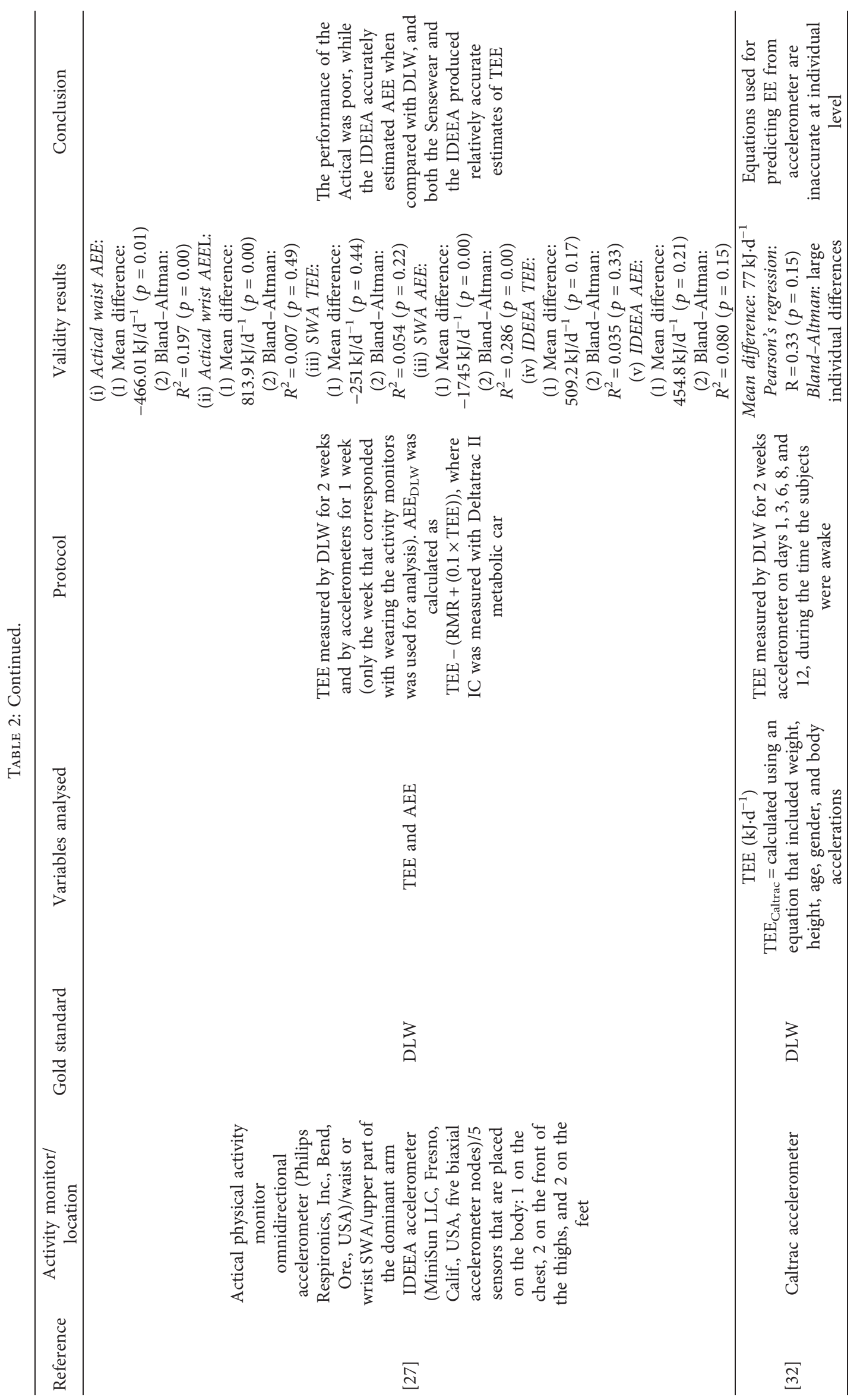




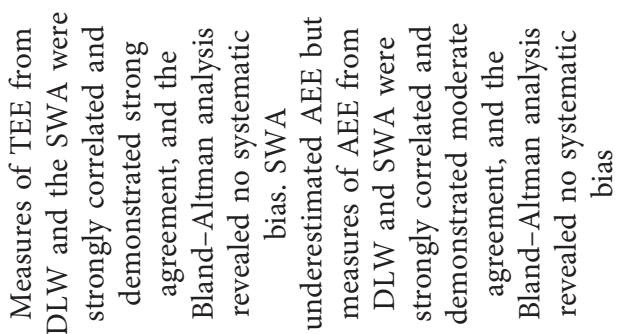

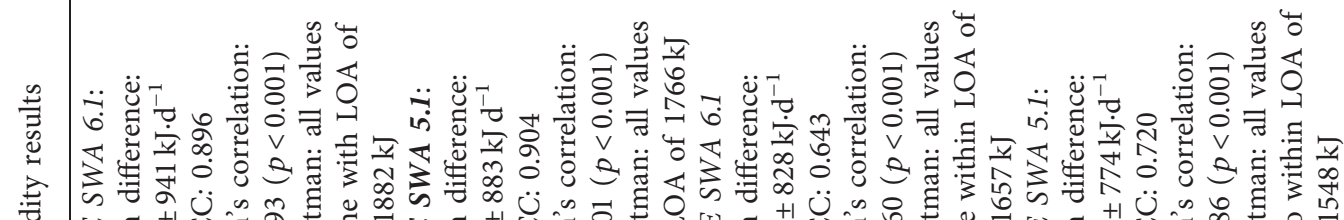

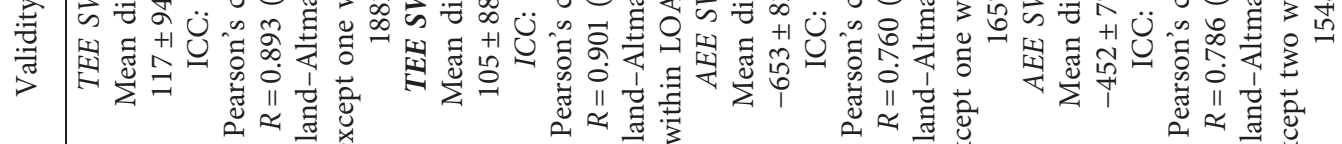

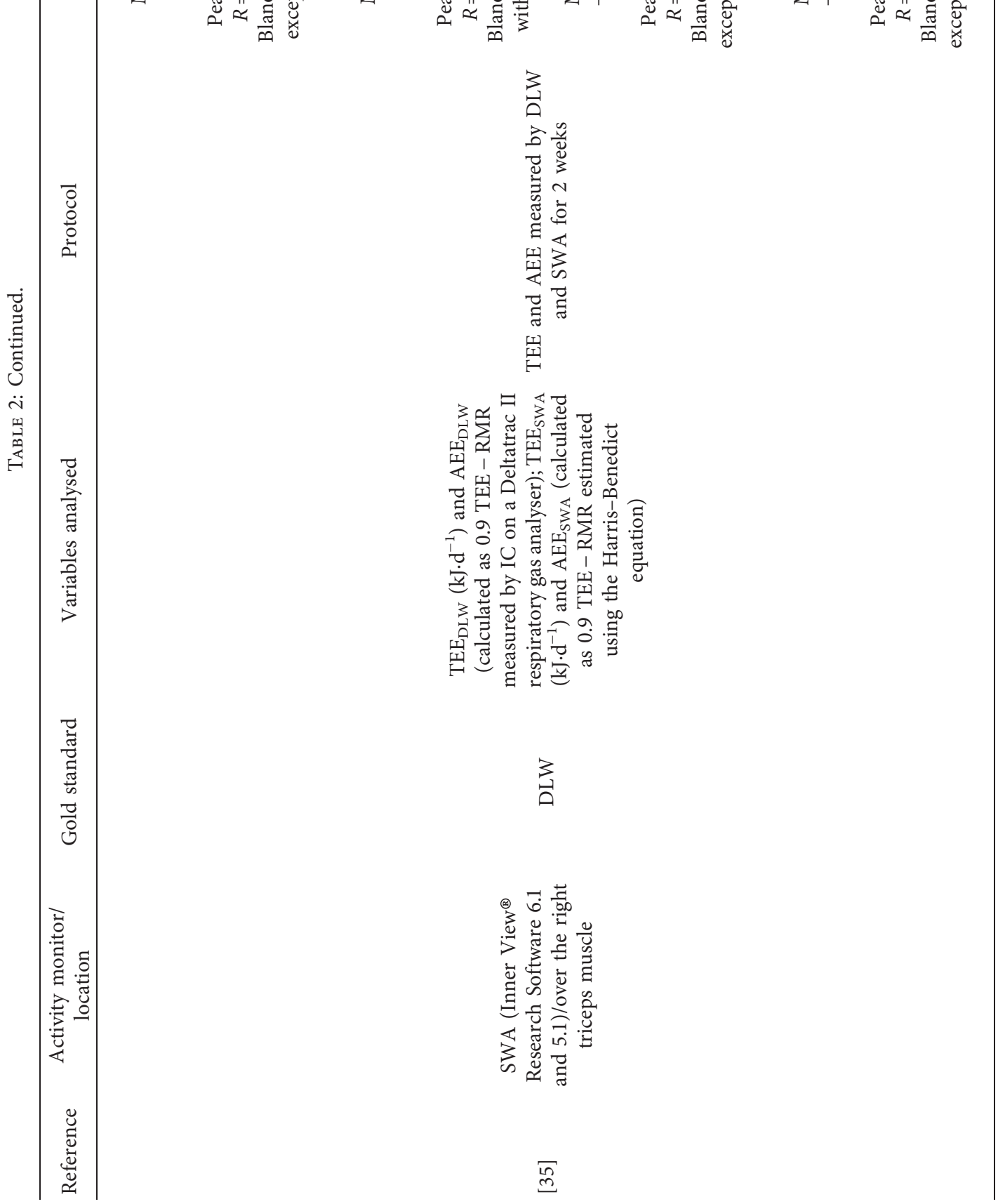




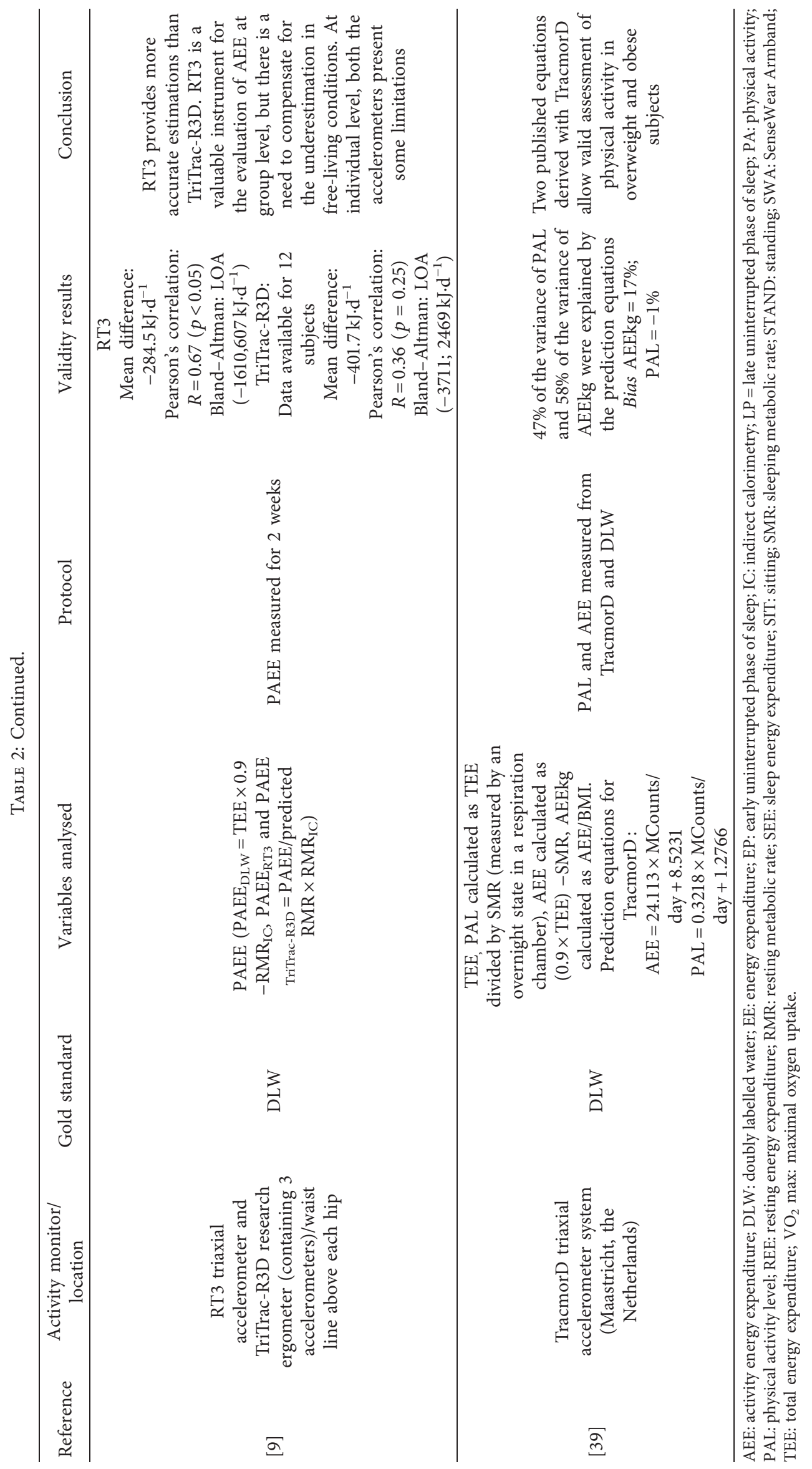


treadmill). The overestimation of SWA was significant, and the Bland-Altman plots showed no agreement for all the 3 physical activities [36].

A more recent version of SWA (Inner View Research Software 6.1) was used in the study of Erdogan et al. The considered exercise consisted of rowing for 10 minutes at two different intensities: authors obtained a good agreement between SWA and IC for high-intensity exercise, but the overestimation of SWA was significant when rowing at moderate intensity [31]. Correlation coefficients indicated a significant association with the gold standard for both the intensities.

Lastly, in the study of Bhammar et al., the SWA's versions 7.0 and 8.1 were validated for the measurements of EE during a structured and a semistructured routine, including a range of activities from light to vigorous intensity. In the structured routine, both the versions of SWA provided a significant overestimation of EE. On the other hand, in the semistructured routine, the most updated version of the software provided estimates not significantly different than the gold standard, as confirmed by the narrow limits of agreements in the Bland-Altman plot.

In the study of Jacobi et al., two different experiments were performed. In experiment 2 , the validity of a triaxial accelerometer (RT3) and the validity of TriTrac-R3D (in which 3 accelerometers are incorporated) were compared with IC for walking at 3 different speeds. In addition, the validity of the same accelerometers (RT3 and TriTrac-R3D) in estimating PAEE was evaluated against DLW in a group of 13 overweight or obese women in free-living conditions (experiment 1, see Table 1 and Table 2) [9]. Regarding experiment 2, both devices showed a trend toward overestimation, but RT3 measures were more accurate. Despite the better results obtained by RT3, authors specified that this accelerometer presents some limitations when the individual level is considered, as shown by Bland-Altman plots [9].

Dondzila and Garner evaluated the accuracy of the consumer-grade accelerometer Fitbit Charge 3-axis during walking and jogging: an important underestimation was obtained for both the activities, suggesting a low reliability of the device [28].

In the study of Imboden et al., the validity of the research-grade accelerometer ActiGraph GT3X was tested in a semistructured routine, including both sedentary and ambulatory/cycling activities. A large underestimation of PAEE was observed, mostly driven by the household activities included in the routine [33].

Machač et al. enrolled adult volunteers with type I or type II diabetes, in order to verify the accuracy of accelerometers in this specific population. The mean BMI of the participants indicated that they were, as a group, obese. The protocol consisted of 3 sessions (15 minutes each) of walking on a treadmill. The SWA (software version 6.1) provided accurate estimates at different speeds (as demonstrated by Pearson's correlations between 0.63 and 0.79 ). Based on the authors' interpretation, the positive results were due to the reasonable duration of the protocol, considering that shorter experiments are more prone to bias [34].

In the study of Swartz et al., participants were divided into 3 categories based on BMI (normal weight, overweight, and obese), and results were presented for all the samples and by BMI group. The accelerometer Kenz Lifecorder EX was validated during a 6-stage walking protocol on a treadmill. In overweight and obese participants, there was a trend toward overestimation, and in general, the authors concluded that the instrument was not accurate for measuring EE in individuals with varying BMI [37].

The study of Thorp et al. was the only one to consider sedentary behaviour, defined as any waking behaviour characterized by an EE of 1.5 or fewer METs while sitting, reclining, or lying and including most office work, driving a car, standing quietly, and sitting while watching television [38]. In this study, the SWA software version 7.0 was evaluated. Bland-Altman plots showed a moderate agreement between the two methods when sitting, and a strong agreement when standing, while correlation coefficients indicated a significant association with the gold standard for both standing and sitting.

3.8. TEE and PAEE under Free-Living Conditions. Five included studies performed Bland-Altman analysis for comparing EE measured by IC and accelometers [9, 27, 32, $35,41]$.

In the study of Fogelholm et al., the accuracy of the Caltrac uniaxial accelerometer in the measurement of TEE was evaluated: even if the accuracy of the instrument was good at a group level, at individual level differences were large [32].

An underestimation of EE in free-living conditions was obtained by the work of Jacobi et al. RT3 limits of agreement were smaller than TriTrac-R3D, but presented limitations at individual levels [9].

Correa et al. selected a subsample of participants from 2 clinical trials aimed to weight loss and evaluated the validity of 3 different accelerometers for the measurement of AEE and TEE under free-living conditions. In contrast with other studies included in the review $[9,32]$, two accelerometers provided accurate estimates. More specifically, Bland-Altman plots showed that SWA and IDEEA accurately estimated TEE, and the IDEEA accurately measured AEE. On the other hand, the performance of Actical was low. Authors stated that the study provides a modest support for the assertion that multisensor activity monitors produce more accurate estimates of AEE and TEE, compared with traditional accelerometers; however, they also expressed the need for further validation research [27].

In line with the findings of Correa et al., the study of Mackey et al. tested the accuracy of TEE and AEE estimates of the SWA, using software versions 6.1 and 5.1 in a sample of older participants (78-89 years old), which were overweight as a group. Both versions showed high Pearson's correlation coefficients $(R>0.75)$ for TEE. On the other hand, AEE was underestimated by both versions 6.1 and 5.1. 
Nevertheless, Bland-Altman plots revealed no systematic bias when considering both TEE and AEE [35].

Finally, Valenti et al. enrolled obese and overweight individuals in order to validate a new specific equation for the estimation of TEE from TracmorD accelerometer under free-living conditions. The developed equation allowed valid assessment of physical activity level (PAL, calculated as TEE/ sleeping metabolic rate) and AEE/body weight (AEEkg). More specifically, PAL estimates were highly correlated with the gold standard measurements $(R=0.69)$, and the errors were correlated with PAL but not with BMI. Similarly, AEEkg and predictions from the new equation were highly correlated $(R=0.76, p<0.001)$ and the errors did not correlate with the BMI [39].

\section{Discussion}

Despite the numerous studies aimed to validate the use of accelerometers in estimating EE, there are no systematic reviews that focus on the validity of these devices in overweight and/or obese individuals.

The use of accelerometers in this population presents some potential issues. For instance, for the same physical activity effort, obese/overweight subjects spend more energy than normal-weight individuals [42], due to the increased fatness. In fact, the physiological energy expenditure is influenced by both body weight and movement efficiency and so may not necessarily reflect the intensity and amount of body movement [43]. In addition, the accuracy of accelerometers is reduced if the sensor is positioned at an angle, which may happen more often in overweight or obese individuals $[43,44]$, due to the increased fat mass. Moreover, it has been demonstrated that the error of accelerometers' estimates is affected by the activity type and intensity (being higher in the case of vigorous activities and sedentary behaviors) and by differences in body weight, with increasing BMI being associated with increased bias $[45,46]$. Furthermore, some accelerometers, such as SWA, require the insertion of subjects' characteristics (gender, sex, and smoking status) in the equations, including anthropometric parameters, whose measurement itself can represent a source of bias, especially for the estimation of REE [14].

In our review, accelerometers' estimations of $\mathrm{EE}$ in obese and overweight individuals are shown to be inaccurate in many studies and most authors advise to improve the accuracy of algorithms for the SWA software or the predicted equations for the other accelerometers.

Even though results on the validity of accelerometers in obese and overweight individuals remain contradictory, it is possible to notice a trend toward overestimation for REE (see Supplementary Material 1), which was measured by SWA in the included studies.

The findings on REE obtained in our review are in contrast with those obtained in the normal-weight population. In the study of Malavolti et al., SWA (software 4.0) provided accurate estimates of the REE, not significantly different than those of the IC, as confirmed by the Bland-Altman plot [17]. Similarly, Casiraghi et al. obtained a high Pearson's correlation $(R=0.95)$ when testing the accuracy of SWA (software 6.1) for measuring REE in healthy normal-weight individuals [47]. In addition, Zorrilla-Revilla et al. found small mean absolute percentage error (MAPE) and percentage mean change in healthy adults, when measuring RMR using the SWA mini (software 7.0) [14].

In our review, a similar trend toward overestimation was obtained also for PAEE, compared with IC (see Supplementary Material 1).

The triaxial accelerometer RT3 seemed to be more accurate than the uniaxial accelerometers for predicting EE in obese and overweight individuals during walking on a treadmill [9]. This is in line with results obtained in the general population, which show an accurate measure of PAEE by the RT3, both under laboratory and the free-living conditions [48, 49].

A previous version of SWA (4.0) was assessed by Papazoglou et al. in obese individuals, during an activity protocol including pedalling, stepping, and walking. Authors recommended to improve the accuracy of the software, though the study was published in 2006 and an old version has been used.

Two of the included studies came to an opposite conclusion when evaluating the accuracy of the more recent software version $6.1[31,34]$. On the one hand, Erdogan et al. claimed the need to improve the accuracy of the SWA algorithms for obese individuals, and on the other hand, Machač et al. obtained a good accuracy in obese volunteers with diabetes. Regarding the corresponding findings in healthy adults, SWA 6.1 outperformed other activity monitors for the estimation of EE during light- to moderateintensity semistructured activities [50].

Swartz et al. validated the Kenz Lifecorder accelerometer in volunteers with various BMI (normal weight, overweight, and obese): the accelerometer was judged as inaccurate due to the important overestimation. This result is in line with evidences obtained in normal-weight adults, which show an overestimation of the Kenz Lifecorder at some walking speeds [51].

In one of the included studies, the recent software version of SWA (8.1) showed a better performance than SWA 7.1 in a semistructured routine, which reflected until a certain level the free-living conditions [26]. In agreement with this finding, in a study in which participants were of normal-weight and completed a series of physical exercises, the SWA version 8.1 outperformed the previous one, showing a MAPE of $20 \%$ in total [50].

In the included study of Thorp et al. on sedentary behaviours, SWA 8.1 showed a good accuracy when standing, but not when sitting [38]. However, also a study enrolling normal-weight participants obtained an underestimation of SWA during office work (standing or sitting) [53].

In one of the included studies, Imboden et al. found an important underestimation of PAEE during a semistructured routine by the accelerometer ActiGraph GT3X. The same accelerometer proved to be a good tool to predict $\mathrm{EE}$ in normal-weight adults during walking, compared with IC, and under free-living conditions, compared with DLW 
$[54,55]$. Interestingly, in the study of Imboden et al., the household activities presented the highest bias [33].

The Fitbit Charge was considered not reliable for the measurement of EE in a walking protocol [28]. In line with this finding, the Fitbit Charge showed to overestimate moderate to vigorous physical activity in normal-weight participants, compared with other research-grade accelerometers [56].

Regarding the measurement of TEE in free-living conditions, two studies obtained important differences at the individual level $[9,32]$. It should be specified that in these two studies, only overweight/obese participants were enrolled. The findings related to this population were in contrast with those in healthy subjects [16].

On the other hand, other studies highlighted the potential of the multisensor accelerometers (SWA and IDEEA), compared with traditional accelerometers, finding a good level of agreement with the DLW [27, 35]. However, differences were still large at the individual level.

In the study of Valenti et al., equations developed by the authors specifically for the obese population improved the accuracy of EE predictions, when using the accelerometer TracmorD [39].

We must note two limitations inherent in this systematic review. Firstly, our search method and inclusion criteria have restricted the number of included studies. Secondly, the selected studies varied greatly in population characteristics, accelerometer models and algorithms, and protocols, making it difficult to compare the obtained results. Due to the heterogeneity of the included studies, a meta-analysis was not possible. In addition, the indirect calorimetry is currently considered the most accurate approach for estimating EE in obese individuals and the only accurate approach in extreme obese patients (class III) [5]. On the other hand, DLW is the most accurate and objective measurement for assessing PA in free-living individuals [57], but a greater underestimation of EE has been shown in heavier and fatter subjects [58]. Although the possible underestimation in this specific population should be taken into account, it still remains more accurate than other methods of PA assessment in free-living conditions [57].

For the measurement of REE and PAEE, the different protocols followed in the included works (i.e., hours of fasting and hours of abstention from alcohol and physical activity before the test and duration of the test) reflect the need to publish a standardized protocol for validation studies, with the aim to facilitate the comparison of results. In most of the included studies, exclusion criteria were based on the presence of disease conditions (such as chronic organ disease, cardiovascular disease, and cancer) or medical conditions that could interfere with the execution of the physical exercises. We believe that, in addition, the use of medications known for influencing the thermoregulatory process (e.g., sibutramine, anticholinergics, psychotrops) or that may interfere with energy expenditure (beta-adrenergic and corticosteroids) should be always taken into consideration [31]. We also recommend an abstention from food and caffeine (minimal 4 hours) and moderate-vigorous physical activity (minimal 2 hours). In any case, even when the abstention from physical exercise is not indicated in the protocol, laboratory experiments should be initiated after a period of rest (10-20 minutes) in order to minimize possible effects of recent physical activities such as dressing, driving, or walking [59, 60]. Furthermore, taking into account the well-known effects of smoking in affecting EE [61, 62] and that smoking can affect the measurement of RMR in obese patients [63], smokers should be excluded from the sample population, or the results should be presented considering the smoking status. This exclusion could be avoided in the case of accelerometers that take into account the smoking status in the equations (i.e., SWA) [34].

Once enough studies specific for obese and overweight individuals with standardized protocols will be available, the establishment of correction factors for accelerometer estimations will be possible. It is important to specify that, even if accelerometers do not allow us to correctly evaluate the exact energy balance in overweight or obese individuals, they can be very useful in clinical practice, as observed for supervised physical activity, for the monitoring of behavioural changes and in the consequent motivational stimulus to undertake more active lifestyles.

$\begin{array}{ll}\text { Abbreviations } \\ \text { AEE: } & \text { Activity EE } \\ \text { BMI: } & \text { Body mass index } \\ \text { DLW: } & \text { Doubly labelled water } \\ \text { EE: } & \text { Energy expenditure } \\ \text { MAPE: } & \text { Mean absolute percentage error } \\ \text { PA: } & \text { Physical activity } \\ \text { PAEE: } & \text { Physical activity energy expenditure } \\ \text { QUADAS-2: } & \text { Quality assessment of diagnostic accuracy } \\ & \text { studies } \\ \text { REE: } & \text { Resting energy expenditure } \\ \text { RMR: } & \text { Resting metabolic rate } \\ \text { SEE: } & \text { Sleep energy expenditure } \\ \text { SWA: } & \text { SenseWear Armband } \\ \text { TEE: } & \text { Total energy expenditure } \\ \text { VO }{ }_{2} \text { max: } & \text { Maximal oxygen uptake. }\end{array}$

\section{Disclosure}

Where authors are identified as personnel of the International Agency for Research on Cancer/World Health Organization, the authors alone are responsible for the views expressed in this article and they do not necessarily represent the decisions, policy, or views of the International Agency for Research on Cancer/World Health Organization.

\section{Conflicts of Interest}

The authors declare no competing financial interests.

\section{Authors' Contributions}

S.P. and A.D. devised the research strategy and the initial screening. S.P. and A.D. extracted the data which were checked by A.L. Disagreements were resolved by consensus and by reference to F.V. All authors contributed to the data 
synthesis and production of the paper. All authors read and approved the final manuscript.

\section{Supplementary Materials}

Figure S1: Pearson's correlation/regression between accelerometer and indirect calorimetry estimates of resting energy expenditure (REE). Each horizontal bar represents the coefficient ( $R$ value). Figure S2: Pearson's correlation/ regression between accelerometer and indirect calorimetry estimates of energy expenditure during different physical exercises in laboratory conditions. Each horizontal bar represents the coefficient ( $R$ value). Figure S3: Pearson's correlation/regression between the accelerometer and doubly labelled water estimates of energy expenditure under free-living conditions (AEE=activity energy expenditure; $\mathrm{TEE}=$ total energy expenditure). Each horizontal bar represents the coefficient ( $R$ value). Figure S4: mean difference between accelerometer and indirect calorimetry (IC) estimates of resting energy expenditure (REE) (expressed in kJ/day). Figure S5: mean difference between accelerometer and indirect calorimetry estimates of energy expenditure during different physical exercises under laboratory conditions (expressed in $\mathrm{kJ} / \mathrm{min}$ ). Figure S6: mean difference between accelerometer and the doubly labelled water estimates of the activity energy expenditure (AEE) under free-living conditions (expressed in $\mathrm{kJ} /$ day). Figure S7: mean difference between accelerometer and doubly labelled water estimates of the total energy expenditure (TEE) under free-living conditions (expressed in $\mathrm{kJ} /$ day). (Supplementary Materials)

\section{References}

[1] R. J. Shephard and C. Tudor-Locke, "Physical activity and optimal health: the challenge to epidemiology," in The $\mathrm{Ob}$ jective Monitoring of Physical Activity: Contributions of Accelerometry to Epidemiology, Exercise Science and Rehabilitation, pp. 1-38, Springer, Cham, Switzerland, 2016.

[2] G. D. Foster and B. G. McGuckin, "Estimating resting energy expenditure in obesity," Obesity Research, vol. 9, no. Suppl 5, pp. 367S-372S, 2001.

[3] S. H. Boutcher and S. L. Dunn, "Factors that may impede the weight loss response to exercise-based interventions," Obesity Reviews, vol. 10, no. 6, pp. 671-680, 2009.

[4] P. T. Katzmarzyk and S. A. Lear, Physical Activity for Obese Individuals: A Systematic Review of Effects on Chronic Disease Risk Factors, Centre for Reviews and Dissemination (UK), York, UK, 2012, https://www.ncbi.nlm.nih.gov/books/ NBK84704/ Database of Abstracts of Reviews of Effects (DARE): Quality-Assessed Reviews [Internet].

[5] H. A. Haugen, L.-N. Chan, and F. Li, "Indirect calorimetry: a practical guide for clinicians," Nutrition in Clinical Practice, vol. 22, no. 4, pp. 377-388, 2007.

[6] A. P. Hills, N. Mokhtar, and N. M. Byrne, "Assessment of physical activity and energy expenditure: an overview of objective measures," Frontiers in Nutrition, vol. 1, 2014.

[7] R. D. Gupta, R. Ramachandran, P. Venkatesan, S. Anoop, M. Joseph, and N. Thomas, "Indirect calorimetry: from bench to bedside," Indian Journal of Endocrinology and Metabolism, vol. 21, no. 4, pp. 594-599, 2017.
[8] K. L. Campbell, P. R. E. Crocker, and D. C. McKenzie, "Field evaluation of energy expenditure in women using tritrac accelerometers," Medicine \& Science in Sports \& Exercise, vol. 34, no. 10, pp. 1667-1674, 2002.

[9] D. Jacobi, A.-E. Perrin, N. Grosman et al., "Physical activityrelated energy expenditure with the RT3 and TriTrac accelerometers in overweight adults*," Obesity, vol. 15, no. 4, pp. 950-956, 2007.

[10] C.-C. Yang and Y.-L. Hsu, "A review of accelerometry-based wearable motion detectors for physical activity monitoring," Sensors, vol. 10, no. 8, pp. 7772-7788, 2010.

[11] G. Plasqui, "Smart approaches for assessing free-living energy expenditure following identification of types of physical activity," Obesity Reviews: An Official Journal of the International Association for the Study of Obesity, vol. 18, no. Suppl 1, pp. 50-55, 2017.

[12] D. Andre, R. Pelletier, J. Farringdon et al., "The development of the SenseWear armband, a revolutionary energy assessment device to assess physical activity and lifestyle," 2006.

[13] P. H. Lee, Y. Yu, I. McDowell, G. M. Leung, T. Lam, and S. M. Stewart, "Performance of the international physical activity questionnaire (short form) in subgroups of the Hong Kong Chinese population," International Journal of Behavioral Nutrition and Physical Activity, vol. 8, no. 1, p. 81, 2011.

[14] G. Zorrilla-Revilla, A. Mateos, O. Prado-Nóvoa, M. VidalCordasco, and J. Rodríguez, "Carrying loads: validating a portable tri-axial accelerometer during frequent and brief physical activity," Journal of Science and Medicine in Sport, vol. 20, no. 8, pp. 771-776, 2017.

[15] M. A. Calabró, G. J. Welk, and J. C. Eisenmann, "Validation of the SenseWear pro armband algorithms in children," Medicine \& Science in Sports \& Exercise, vol. 41, no. 9, pp. 17141720, 2009.

[16] D. L. Johannsen, M. A. Calabro, J. Stewart, W. Franke, J. C. Rood, and G. J. Welk, "Accuracy of armband monitors for measuring daily energy expenditure in healthy adults," Medicine \& Science in Sports \& Exercise, vol. 42, no. 11, pp. 2134-2140, 2010.

[17] M. Malavolti, A. Pietrobelli, M. Dugoni et al., "A new device for measuring resting energy expenditure (REE) in healthy subjects," Nutrition, Metabolism and Cardiovascular Diseases, vol. 17, no. 5, pp. 338-343, 2007.

[18] W. J. Tucker, D. M. Bhammar, B. J. Sawyer, M. P. Buman, and G. A. Gaesser, "Validity and reliability of Nike + Fuelband for estimating physical activity energy expenditure," BMC Sports Science, Medicine and Rehabilitation, vol. 7, no. 1, p. 14, 2015.

[19] J. M. Jakicic, M. Marcus, K. I. Gallagher et al., "Evaluation of the SenseWear pro armband??? to assess energy expenditure during exercise," Medicine \& Science in Sports \& Exercise, vol. 36, no. 5, pp. 897-904, 2004.

[20] M. L. Fruin and J. W. Rankin, "Validity of a multi-sensor armband in estimating rest and exercise energy expenditure," Medicine \& Science in Sports \& Exercise, vol. 36, no. 6, pp. 1063-1069, 2004.

[21] K. Y. Chen, S. A. Acra, C. L. Donahue, M. Sun, and M. S. Buchowski, "Efficiency of walking and stepping: relationship to body fatness," Obesity Research, vol. 12, no. 6, pp. 982-989, 2004.

[22] W. R. Leonard, "Laboratory and field methods for measuring human energy expenditure," American Journal of Human Biology, vol. 24, no. 3, pp. 372-384, 2012. 
[23] D. Ndahimana and E.-K. Kim, "Measurement methods for physical activity and energy expenditure: a review," Clinical Nutrition Research, vol. 6, no. 2, pp. 68-80, 2017.

[24] P. F. Whiting, A. W. S. Rutjes, M. E. Westwood et al., "QUADAS-2: a revised tool for the quality assessment of diagnostic accuracy studies," Annals of Internal Medicine, vol. 155, no. 8, pp. 529-536, 2011.

[25] S. Bertoli, A. Posata, A. Battezzati, A. Spadafranca, G. Testolin, and G. Bedogni, "Poor agreement between a portable Armband and indirect calorimetry in the assessment of resting energy expenditure," Clinical Nutrition, vol. 27, no. 2, pp. 307-310, 2008.

[26] D. M. Bhammar, B. J. Sawyer, W. J. Tucker, J.-M. Lee, and G. A. Gaesser, "Validity of SenseWear ${ }^{\circledR}$ armband v5.2 and v2.2 for estimating energy expenditure," Journal of Sports Sciences, vol. 34, no. 19, pp. 1830-1838, 2016.

[27] J. B. Correa, J. W. Apolzan, D. N. Shepard, D. P. Heil, J. C. Rood, and C. K. Martin, "Evaluation of the ability of three physical activity monitors to predict weight change and estimate energy expenditure," Applied Physiology, Nutrition, and Metabolism, vol. 41, no. 7, pp. 758-766, 2016.

[28] C. Dondzila and D. Garner, "Comparative accuracy of fitness tracking modalities in quantifying energy expenditure," Journal of Medical Engineering \& Technology, vol. 40, no. 6, pp. 325-329, 2016.

[29] U. Elbelt, T. Schuetz, I. Hoffmann, M. Pirlich, C. J. Strasburger, and H. Lochs, "Differences of energy expenditure and physical activity patterns in subjects with various degrees of obesity," Clinical Nutrition, vol. 29, no. 6 , pp. 766-772, 2010.

[30] U. Elbelt, T. Schuetz, and L. Herbert, "Estimating resting energy expenditure with a portable armband device in an ambulatory setting," Nutrition in Clinical Practice, vol. 27, no. 6, pp. 825-831, 2012.

[31] A. Erdogan, C. Cetin, H. Karatosun, and L. B. Metin, “Accuracy of the polar S810i(TM) heart rate monitor and the sensewear pro armband(TM) to estimate energy expenditure of indoor rowing exercise in overweight and obese individuals," Journal of Sports Science \& Medicine, vol. 9, no. 3, pp. 508-516, 2010.

[32] M. Fogelholm, H. Hiilloskorpi, R. Laukkanen, P. Oja, W. Van Marken Lichtenbelt, and K. Westerterp, "Assessment of energy expenditure in overweight women," Medicined Science in Sports \& Exercise, vol. 30, no. 8, pp. 1191-1197, 1998.

[33] M. T. Imboden, M. B. Nelson, L. A. Kaminsky, and A. H. Montoye, "Comparison of four Fitbit and jawbone activity monitors with a research-grade ActiGraph accelerometer for estimating physical activity and energy expenditure," British Journal of Sports Medicine, vol. 52, no. 13, pp. 844-850, 2018.

[34] S. Machač, M. Procházka, J. Radvanský, and K. Slabý, "Validation of physical activity monitors in individuals with diabetes: energy expenditure estimation by the multisensor SenseWear armband Pro3 and the step counter omron HJ-720 against indirect calorimetry during walking," Diabetes Technology \& Therapeutics, vol. 15, no. 5, pp. 413-418, 2013.

[35] D. C. Mackey, T. M. Manini, D. A. Schoeller et al., "Validation of an armband to measure daily energy expenditure in older adults," The Journals of Gerontology Series A: Biological Sciences and Medical Sciences, vol. 66A, no. 10, pp. 1108-1113, 2011.
[36] D. Papazoglou, G. Augello, M. Tagliaferri et al., "Evaluation of a multisensor armband in estimating energy expenditure in obese individuals*," Obesity, vol. 14, no. 12, pp. 2217-2223.

[37] A. M. Swartz, S. J. Strath, N. E. Miller et al., "Validity of physical activity monitors in assessing energy expenditure in normal, overweight, and obese adults," The Open Sports Sciences Journal, vol. 2, no. 1, pp. 58-64, 2009.

[38] A. A. Thorp, B. A. Kingwell, C. English et al., "Alternating sitting and standing increases the workplace energy expenditure of overweight Adults," Journal of Physical Activity and Health, vol. 13, no. 1, pp. 24-29, 2016.

[39] G. Valenti, S. G. J. A. Camps, S. P. M. Verhoef, A. G. Bonomi, and K. R. Westerterp, "Validating measures of free-living physical activity in overweight and obese subjects using an accelerometer," International Journal of Obesity, vol. 38, no. 7, pp. 1011-1014, 2014.

[40] R. Waldburger, R. Zazai, B. Wilms, B. Ernst, M. Thurnheer, and B. Schultes, "Resting energy expenditure values assessed by a multi-sensor armband show a low accuracy in obese subjects," e-SPEN Journal, vol. 8, no. 6, pp. e246-e250, 2013.

[41] F. Slinde, F. Bertz, A. Winkvist, L. Ellegård, H. Olausson, and H. K. Brekke, "Energy expenditure by multisensor armband in overweight and obese lactating women validated by doubly labeled water," Obesity, vol. 21, no. 11, pp. 2231-2235, 2013.

[42] V. P. Lopes, P. Magalhães, J. Bragada, and C. Vasques, "Actigraph calibration in obese/overweight and type 2 diabetes mellitus middle-aged to old adult patients," Journal of Physical Activity and Health, vol. 6, no. s1, pp. S133-S140, 2009.

[43] K. Corder, S. Brage, and U. Ekelund, "Accelerometers and pedometers: methodology and clinical application," Current Opinion in Clinical Nutrition and Metabolic Care, vol. 10, no. 5, pp. 597-603, 2007.

[44] C. Maffeis, Y. Schutz, F. Schena, M. Zaffanello, and L. Pinelli, "Energy expenditure during walking and running in obese and nonobese prepubertal children," The Journal of Pediatrics, vol. 123, no. 2, pp. 193-199, 1993.

[45] K. Koehler and C. Drenowatz, "Monitoring energy expenditure using a multi-sensor device-applications and limitations of the SenseWear armband in athletic populations," Frontiers in Physiology, vol. 8, 2017.

[46] L. G. Sylvia, E. E. Bernstein, J. L. Hubbard, L. Keating, and E. J. Anderson, "Practical guide to measuring physical activity," Journal of the Academy of Nutrition and Dietetics, vol. 114, no. 2, pp. 199-208, 2014.

[47] F. Casiraghi, R. Lertwattanarak, L. Luzi et al., "Energy expenditure evaluation in humans and non-human primates by SenseWear Armband. Validation of energy expenditure evaluation by SenseWear Armband by direct comparison with indirect calorimetry," PLoS One, vol. 8, no. 9, 2013.

[48] J. Vanhelst, L. Béghin, P. Rasoamanana et al., "Calibration of the RT3 accelerometer for various patterns of physical activity in children and adolescents," Journal of Sports Sciences, vol. 28, no. 4, pp. 381-387, 2010.

[49] R. Maddison, Y. Jiang, S. Vander Hoorn et al., "Estimating energy expenditure with the RT3 triaxial accelerometer," Research Quarterly for Exercise and Sport, vol. 80, no. 2, pp. 249-256, 2009.

[50] M. A. Calabró, J.-M. Lee, P. F. Saint-Maurice, H. Yoo, and J. W. Gregory, "Validity of physical activity monitors for assessing lower intensity activity in adults," The International Journal of Behavioral Nutrition and Physical Activity, vol. 11, Article ID 119, 2014. 
[51] M. G. Abel, J. C. Hannon, K. Sell, T. Lillie, G. Conlin, and D. Anderson, "Validation of the Kenz Lifecorder EX and ActiGraph GT1M accelerometers for walking and running in adults," Applied Physiology, Nutrition, and Metabolism, vol. 33, no. 6, pp. 1155-1164, 2008.

[52] G. A. Lopez, J. C. Brønd, L. B. Andersen, M. Dencker, and D. Arvidsson, "Validation of SenseWear armband in children, adolescents, and adults," Scandinavian Journal of Medicine \& Science in Sports, vol. 28, no. 2, pp. 487-495, 2018.

[53] J. Reece, V. Barry, D. Fuller, and J. Caputo, "Validation of the SenseWear armband as a measure of sedentary behavior and light activity," Journal of Physical Activity \& Health, vol. 12, no. 9, 2014.

[54] A. Santos-Lozano, A. Hernández-Vicente, R. Pérez-Isaac et al., "Is the SenseWear armband accurate enough to quantify and estimate energy expenditure in healthy adults?" Annals of Translational Medicine, vol. 5, no. 5, 2017.

[55] A. K. Chomistek, C. Yuan, C. E. Matthews et al., "Physical activity assessment with the ActiGraph GT3X and doubly labeled water," Medicine \& Science in Sports \& Exercise, vol. 49, no. 9, pp. 1935-1944, 2017.

[56] K. J. DeShaw, L. Ellingson, Y. Bai, J. Lansing, M. Perez, and G. Welk, "Methods for activity monitor validation studies: an example with the Fitbit charge," Journal for the Measurement of Physical Behaviour, vol. 1, no. 3, pp. 130-135, 2018.

[57] L. Beechy, J. Galpern, A. Petrone, and S. K. Das, "Assessment tools in obesity-psychological measures, diet, activity, and body composition," Physiology \& Behavior, vol. 107, no. 1, pp. 154-171, 2012.

[58] E. Ravussin, I. T. Harper, R. Rising, and C. Bogardus, "Energy expenditure by doubly labeled water: validation in lean and obese subjects," American Journal of Physiology-Endocrinology and Metabolism, vol. 261, no. 3, pp. E402-E409, 1991.

[59] C. Compher, D. Frankenfield, N. Keim, L. Roth-Yousey, and Evidence Analysis Working Group, "Best practice methods to apply to measurement of resting metabolic rate in adults: a systematic review," Journal of the American Dietetic Association, vol. 106, no. 6, pp. 881-903, 2006.

[60] B. Redondo and Raquel, "Resting energy expenditure; assessment methods and applications," Nutricion Hospitalaria, vol. 31, no. Suppl 3, pp. 245-254, 2015.

[61] S. Gonseth, L. Dugas, B. Viswanathan et al., "Association between smoking and total energy expenditure in a multicountry study," Nutrition \& Metabolism, vol. 11, Article ID 48, 2014.

[62] J. Audrain-McGovern and N. L. Benowitz, "Cigarette smoking, nicotine, and body weight," Clinical Pharmacology \& Therapeutics, vol. 90, no. 1, pp. 164-168, 2011.

[63] T. Hagedorn, E. Poggiogalle, C. Savina et al., "Indirect calorimetry in obese female subjects: factors influencing the resting metabolic rate," World Journal of Experimental Medicine, vol. 2, no. 3, pp. 58-64, 2012. 